Historic, Archive Document

Do not assume content reflects current scientific knowledge, policies, or practices. 


\section{WHOLESALE PRICE LIST for} 1932-1933

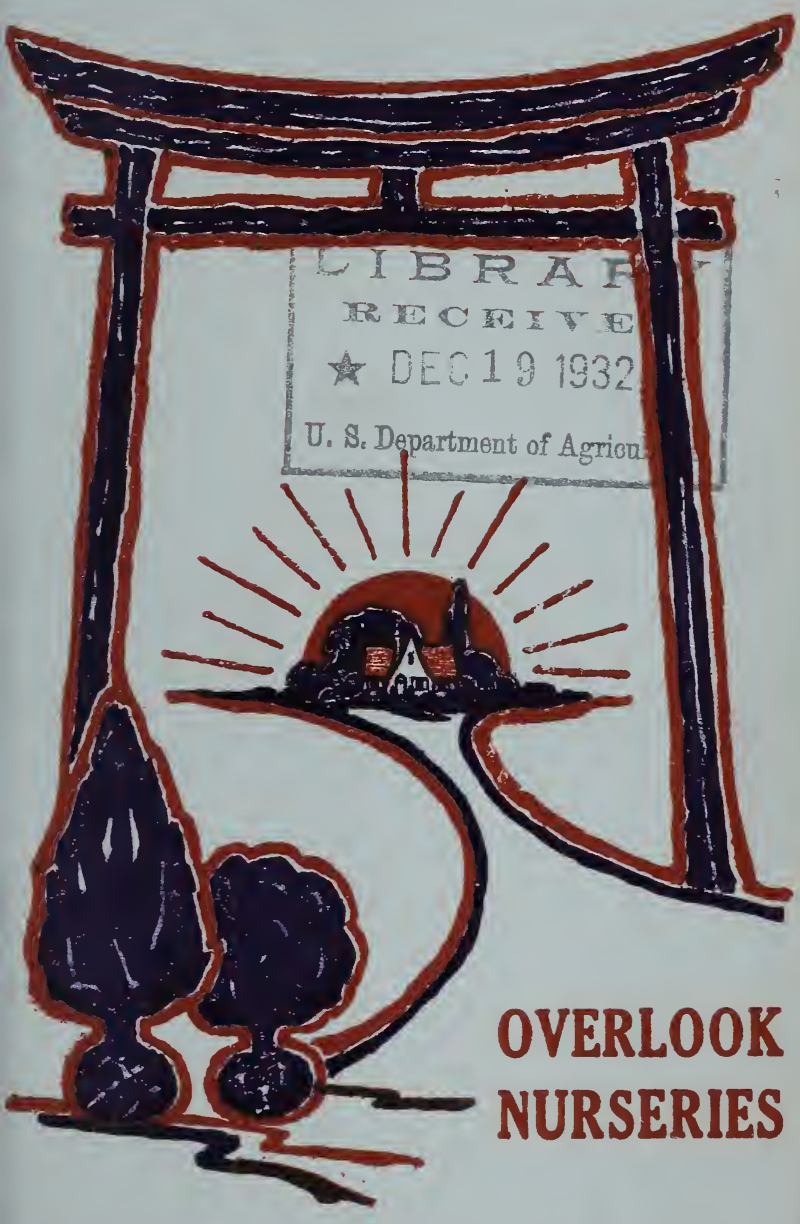

SHIPPING POINT MOBILE AND ORCHARD, ALA.
MAIL ADDRESS CRICHTON, ALA. 


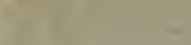

A.

$=$

A.

$$
1=
$$

Wh $1=;=$

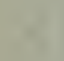

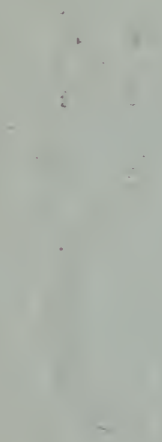

(3)

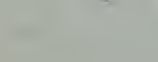

$$
\begin{aligned}
& +\infty x_{x}+\infty \\
& +\infty
\end{aligned}
$$




\title{
Overlook Nurseries
}

S. IMURA

K. SAWADA

\author{
BOX 114, R. F. D. \\ CRICHTON, ALA.
}

Nurseries on Moffat Road, 8 Miles Northeast of Mobile Court House

Shipping Point, Express Shipment, Mobile, Ala. Car-load Shipment, Orchard, Ala.

WHOLESALE PRICE LIST

for

1932-1933

\section{TERMS OF SALE}

TERMS OF PAYMENT-Prices listed here are based on cash payment. Cash with order for immediate shipment. Booking orders will be accepted when accompanied by remittance covering $25 \%$ of purchase amount; the balance to be payable at time shipments demanded. Shipment may be made C. O. D. for balance when one-fourth of the amount of purchase paid with the order. Be sure to include sufficient amount to cover the postage, if you want shipment made by parcel post.

PACKING CHARGES-Are extra at cost for Specimens (B. \& B., Stock). Free of charge for Lining Out Stock .

CAR-LOAD SHIPMENTS-Unusual attractive quotations will be offered on B. \& B. stock for carload orders; speeial discount may be allowed also for B. \& B. Stock delivered at our nursery grounds without boxing.

PRICES APPLIED-5 and 50 of same variety and same size at 10 and 100 rates respectively; make application for 1000 rate. No order of lining out stock less than 5 in same variety and size accepted.

TRANSPORTATION-State whether order is to be shipped by parcel post, express or freight. When no instructions are given, shipment will be made, using our best judgment. Our responsibility ceases upon delivery to forwarding companies; claims for losses or damages must be made upon the latter.

ALL QUOTATIONS are for immediate acceptance, subject to stock being sold. We shall not be subject to any liability should injury befall stock from freeze, fire or any other cause beyond our control.

WE GUARANTEE all stock sent out to be well grown, healthy, true to name and properly packed. Our liability under the foregoing guaranty is limited in amount to original price received. 
CLAIM-If, by any possibility, errors should occur, they will be promptly rectified, if claim is made immediately after the receipt of goods.

REMARKS-Prices listed here are for 10 and 100 rates for Lining Out stock and each for Specimens. Attractive low prices for larger quantities on application.

Lining Out Stock listed here by slender type letters.

(C) indicates "from Cutting", (S) Seedling and (G) Grafting. (T) indicates once transplanted, (TT) twice transplanted, etc. All Lining Out Stock are in bare-roots understood, unless otherwise mentioned.

SPECIMEN STOCK-Listed by bold type letters.- B. \& B. means balled and burlapped. For carload orders or for specimen orders delivered at the field without boxing to your truck special prices most attractive will be offered.

We have several thousands of very large sized splendid specimens, but not listed here. Glad to offer you unusual bargain prices upon your request.

\section{CONIFERS}

\section{CEDRUS DEODARA.}

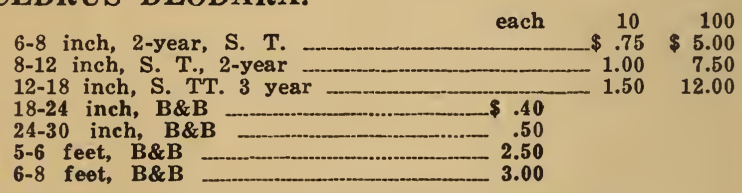

\section{CHAMAECYPARIS LAWSONIA AUREA.}

\section{(Golden Lawson Cypress)}

Brilliant golden color for all seasons and not burns so bad like Alumi and Argentea.

4-6 inch, C. T. 1.00

6-8 inch, C. T.

8-12 inch, G. TT.

\section{CHAM' LAWSONIANA PENDULA GLAUCA.}

\section{(Weeping Lawson Cypress)}

A very beautiful semi-weeping cypress with finely cut blue foliage.

4-6 inch, $\mathrm{C} \mathrm{T}$.

6-8 inch, C. $\mathrm{T}$.

8-12 inch, C. T.

\section{CRYPTOMERIA JAPONICA VAR YOSHINO.} (Very compact growth)

4-6 inch, C. T. 1.00

\section{CUNNINGHAMIA LANCEOLATA.} (Chinese Fir)

6-8 inch, C. T.

8-12 inch, C. T. 
CUPRESSAS ARIZONICA

$\begin{array}{rr}5-7 & \text { feet, } B \& B \\ 7-9 & \text { feet, B\&B } \\ & 1.50 \\ & \end{array}$

CUPRESSAS LUSETANICA (Portuguese Cypress)

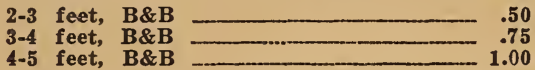

CUPRESSAS SEMPERVIRENSE ROYALI (Italian Cypress)

6-8 inch, C. T.

7.00

8-10 inch, C. T.

2-3 feet, B\&B

3-4 feet, $B \& B$

4-5 feet, $B \& B$

5-6 feet, B\&B

6-8 feet, B\&B

CUPRESSAS TORULOSA MAJESTICA (Bhutan Cypress)

4-6 inch, C. T.

6-8 inch, C. T.

\section{JUNIPERUS CHINENSIS ALBO-VARIEGATA}

(Variegated Chinese Juniper)

6-8 inch, G. TT.

8-10 inch, G. TT.

$12-15$ inch, $B \& B$

15-18 inch, $B \& B$

18-24 inch, $B \& B$

24-30 inch, B\&B

$\begin{array}{r}\hline \\ \hline \\ \hline \\ \hline \\ \hline\end{array} \quad \begin{array}{r}.50 \\ 1.00\end{array}$

\section{JUNIPERUS CHINENSIS COLUMINARIS}

12-15 inch, G. TT.

2-3 feet, B\&B

3-4 feet, B\&B

JUNIPERUS CHINENSIS FEMINA

(Juniperus Sylvestotris)

4-6 inch, C. T.

6-8 inch, C. T.

8-12 inch, C. T.

18-24 inch, B\&B

2-3 feet, $B \& B$

.60

JUNIPERUS CHINENSIS 'MUSCULA

4-6 inch, C. T.

6-8 inch, C. T.

\section{JUNIPERUS CHINENSIS PFITZERIANA}

$\begin{array}{llrr}\text { 4-6 inch, C. T. } & .65 & 5.00 \\ 6-8 \text { inch, C. T. } & .90 & 7.00 \\ 8-12 \text { inch, C. T. } & 1.25 & 9.00 \\ 2-3 \text { feet spread, B\&B } & 1.00 & & \\ 3-4 \text { feet, spread, B\&B - } & 1.50 \\ 4-5 \text { feet spread, B\&B } & 2.00 & & \end{array}$




\section{JUNIPERUS CHINENSIS PROCUMBEN}

(Creeping Juniper)

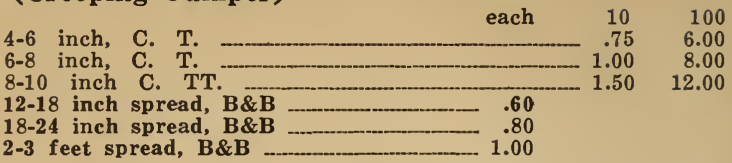

\section{JUNIPERUS CHINENSIS SERGENTI}

(Sergent Trailing Juniper)

2-4 inch C. $\mathrm{T}$.

4-6 inch $\mathrm{C}$. $\mathrm{T}$.

8-10 inch G. TT.

\section{JUNIPERUS COMMUNIS (English Juniper)}

6-8 inch C. T.

8-12 inch C. T.

12-18 inch C. TT.

1.05

18-24 inch B\&B _.

2-3 feet $B \& B$

$5-6$ feet $B \& B$, sheared ___ 1.75

6-8 feet $B \& B$, sheared .___________ 2.50

\section{JUNIPERUS COMMUNIS ASHFORDII}

6-8 inch C. T.

8-12 inch C. $T$.

12-18 inch C. TT.

12.00

18-24 inch $B \& B$

2-3 feet $B \& B$

.35

JUNIPERUS COMMUNIS COLUMNALIS STRICTA

Strictly columnar, just like Royal Italian Cypress. The plant in height of 6-7 feet has only 8-10 inches of diameter in its width without receiving any shearing. Very compact growth with silverly green foliage. The original plant selected by us years ago, out of many thousands of seedlings. We believe this is narrowest columnar juniper of the English type. 4-6 inch C. T.

6-8 inch C. $\mathrm{T}$.

10.00

\section{JUNIPERUS COMMUNIS DEPRESSA}

\section{(Canadian Juniper)}

6-8 inch C. T.

8-12 inch C. $T$.

12-15 inch C. TT.

15-18 inch spread, $B \& B$

18-24 inch spread, B\&B

.35

JUNIPERUS COMMUNIS DEPRESSA AUREA (Golden Canadian Juniper)

6-8 inch C. T.

8-12 inch C. TT.

12-15 inch C. TT.

12.00

15-18 inch spread, B\&B

18-24 inch spread, B\&B

.50

.75 
JUNIPERUS CONFERTA (Shore Juniper)

$\begin{array}{lll} & \text { each } & 10\end{array}$

$12-18$ inch C. TT.

2-3 feet spread, $B \& B$

3-4 feet spread, B\&B 1.25

JUNIPERUS EXCELSA STRICTA

(Spiny Greek Juniper)

4-6 inch C. T.

6-8 inch C. T.

8-10 inch C. TT.

8.00

10-12 inch C. TT.

10.00

12-15 inch C. TT.

12-15 inch, B\&B

12.00

15-18 inch $B \& B$

.30

1.25

JUNIPERUS HORIZONTALIS DOUGLASI

2-4 inch C. T.

4-6 inch C. T.

\section{JUNIPERUS JAPONICA OBLONGA}

4-6 inch C. T.

$12-15$ inch G. TT.

7.00

15-18 inch

18-24 inch $B \& B$

\section{JUNIPERUS VIRGINIANA CANNARTI}

12-18 inch G. TTT.

18-24 inch $B \& B$

2-3 feet $B \& B$

\section{JUNIPERUS ELEGANTISSIMA}

12-18 inch G. TTT.

2-3 feet $B \& B$

3-4 feet $B \& B$

JUNIPERUS VIRGINIANA GLAUCA (Silver Juniper)

12-18 inch G. TTT.

18-24 inch $B \& B$

2-3 feet $B \& B$

3-4 feet $B \& B$

4-5 feet $B \& B$ - 2.00

\section{JUNIPERUS VIRGINIANA KOSTERI}

4-6 inch C. T.

8-12 inch C. TT.

2-3 feet spread, B\&B 1.00

3-4 feet spread, B\&B 1.50

PODOCARPUS SINENSIS MACROPHYLLA

(Large Leaved Japan Yew)

4-6 inch C. T.

8-12 inch C. TT.

3-4 feet $B \& B$

4-5 feet $B \& B$

5-6 feet $B \& B$

6-7 feet $B \& B$ 
$\begin{array}{llll}6-8 & \text { each } & 10 & 100\end{array}$

$8-12$ inch C. T. $\quad .65 \quad 5.00$

RETINOSPORA ERICOIDES (Columnar Cypress)

4-6 inch C. T.

6-8 inch C. T. ___ _ $\quad .80 \quad 6.00$

8-10 inch C. T. W $1.00 \quad 8.00$

RETINOSPORA FILIFERA

(Thread-Leaved Cypress)

6-8 inch C. T.

8-12 inch C. T. _________________ $\quad .90 \quad 7.00$

$12-15$ inch C. TT. - _ _ _ - 1.5012 .00

15-18 inch B\&B B__ $\quad .40$

18-24 inch $B \& B$ -

2-3 feet $B \& B$

RETINOSPORA FILIFERA AUREA

(Golden Thread-Leaved Cypress)

6-8 inch C. T. ___ $\quad .75$

8-12 inch C. T. W $1.25 \quad 9.00$

12-15 inch C. TT. -

RETINOSPORA LEPTOCLADA

4-6 inch C. T.

6-8 inch C. T.

8-12 inch C. TT. _._______ $1.00 \quad 8.00$

12-18 inch B\&B

.40

RETINOSPORA OBTUSA CRIPPSI AUREA (Real Golden Cypress)

4-6 inch C. T.

6-8 inch C. T. 1.00

8-12 inch C. TT. -

$12-15$ inch C. TT. - $1.75 \quad 15.00$

15-18 inch B\&B _.

RETINOSPORA OBTUSA

12-18 inch C. TT.

3-4 feet B\&B

4-5 feet $B \& B$

5-6 feet $B \& B$

RETINOSPORA OBTUSA NANA COMPACTUM

2-4 inch C. T.

10.00

RETINOSPORA PISIFERA AUREA

(Golden Sawara Cypress)

6-8 inch C. T.

8-12 inch C. T.

12-18 inch C. TT.

18-24 inch $B \& B$

7.00

$1.25 \quad 10.00$

2-3 feet $B \& B$

.50

.60

RETINOSPORA PLUMOSA

6-8 inch C. $T$.

8-12 inch C. T.

12-18 inch C. T.

18-24 inch $B \& B$

2-3 feet $B \& B$

3-4 feet $B \& B$

4-5 feet $B \& B$

5-6 feet $B \& B$

$\mathbf{5 0}$
$-\mathbf{6 0}$
-

.75 
RETINOSPORA PLUMOSA AUREA

$\begin{array}{llll} & \text { each } & 10 & 100\end{array}$

6-8 inch C. T.

8-12 inch C. T.

12-18 inch C. $\mathrm{T}$.

18-24 inch $B \& B$

7.00

10.00

2-3 feet $B \& B$

3-4 feet $B \& B$

.40

4-5 feet $B \& B$

.50

.60

.85

RETINOSPORA PLUMOSA ARGENTA

(Silver-tip Dwarf Plume Cypress)

4-6 inch C. T.

6-8 inch C. T.

7.00

8-10 inch C. $T$.

10-15 inch C. TT.

9.00

15-18 inch $B \& B$

RETINOSPORA PLUMOSA GLAUCA

\section{(Beautiful Silvery-Blue Color)}

6-8 inch C. T.

8-12 inch C. TT.

RETINOSPORA SQUARROSA SIEBOLDI (Andely Cypress)

6-8 inch C. T.

8-12 inch C. $T$.

12-15 inch C. TT.

15-18 inch $B \& B$

18-24 inch $B \& B$

\section{RETINOSPORA SQUARROSA VEITCHII}

6-8 inch C. T.

8-12 inch $\mathrm{C}$. $\mathrm{T}$.

12-18 inch C. TT.

18-24 inch $B \& B$

12.00

2-3 feet B\&B

3-4 feet $B \& B$

.40

.60

.75

THUJA ORIENTALIS (Chinese Arborvitea)

6-8 inch $\mathrm{S}$.

8-12 inch $S$.

12-18 inch $\mathrm{S}$

THUJA ORIENTALIS AUREA CONSPICUA

8-12 inch G. TT.

12-18 inch G. TT.

2-3 feet (Grafted) B\&B

1.50

12.00

4-5 feet (Grafted) B\&B

THUJA ORIENTALIS AUREA NANA (Berkman's Golden)

4-6 inch C. T.

6-8 inch

18-24 inch $B \& B$

24-30 inch $B \& B$ 
THUJA ORIENTALIS AUREA GLOBOSA (Golden Ball Arborvitea)

Strictly dwarf, and perfect globe shape with bright golden foliages for all seasons. We originated this valuable variety years ago from a seedling of Conspicua. Very slow grower, but we assure you it is best Arborvitea for urn or pot culture.

2-4 inch C. T. (Spring delivery only) -.-... $1.00 \quad 8.00$

\section{THUJA ORIENTALIS BAKERII}

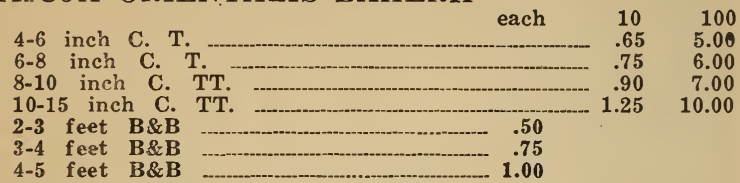

\section{THUJA ORIENTALIS BONITA}

4-6 inch $\mathrm{C}$. $\mathrm{T}$

6-8 inch C. T.

8-10 inch C. TT.

15-18 inch $B \& B$

18-24 inch B\&B

\section{THUJA ORIENTALIS COLUMNALIS STRICTA}

Originated by us. Much narrower than Bakers and decidedly columnar growth with very compact branchlets. This is indeed an ideal plant we have been looking for many years to take place of Italian Cypress which does not thrive on some soil. This is first year we offer in

the market. Limited supply, book order early.

2-4 inch C. T. (Spring delivery only) -... 1.00

8-12 inch G. TT.

\section{THUJA ORIENTALIS GLAUCA}

(Blue-Green Arborvitea).

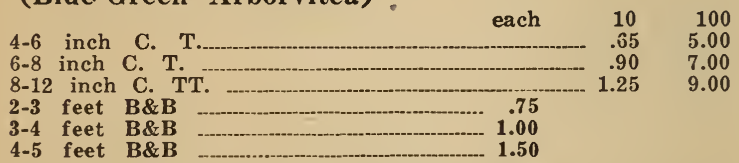

THUJA ORIENTALIS ROSEDALE

4-6 inch C. T.

6-8 inch C. T.

8-12 inch C. TT.

18-24 inch $B \& B$.

$2-3$ feet $B \& B$ - $\quad .60$

$3-4$ feet $B \& B \quad \ldots$

THUJA ORIENTALIS TEXANA GLAUCA

4-6 inch C. T.

6-8 inch C. T.

$1.00 \quad 8.00$

8-12 inch C. TT. 1.00 


\section{BROAD-LEAVED EVERGREENS}

\section{ABELIA GRANDIFLORA}

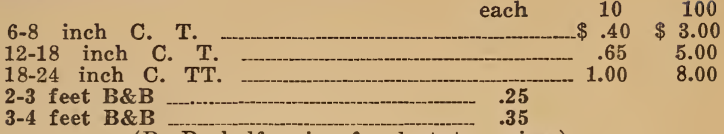

(B. R. half price for last two sizes)

\section{AZALEA (Two new Azaleas Introduced by Us) GULF PRIDE}

A sport of Indica Alba (Ledifolia Alba). Flower medium to large, straight petals of most charming light purple color, could never be seen in other varieties, with four or five flowers clustered like Rhododendron. It blooms at mid-season. Leaves broad and thick, dull green covered with hairy secretion. Rather upright and open growth unless well pinched. Very hardy and stands against cold better than any other Indica varieties.

\section{VIOLA}

A sport of Indica Rosea (Ledifolia Rosea) Character of parent tree faithfully inherited, but the color of the flower that is admirable violet mauve. Very hardy and vigorous grower.

Prices of the above two new Azaleas

4-6 inch $\mathrm{C}$. $\mathrm{T}$.

4-6 inch, $2 \frac{1}{2}$ inch pots

1.75

6-8 inch C. TT., bushy

8-10 inch C. TTT., bushy

4.00

35.00

6.00

50.00

\section{AZALEA INDICA}

\section{PINK AND ROSE RED FLOWERS}

BRILLIANT-Bright water-melon red, flower medium, midseason, small leaves, compact growth.

CROEMINA-Shell pink, large flower, earliest bloomer and very rapid grower, but not compact.

GEORGE FRANC-Flame pink with deep carmine spot near the throat, large flower, early.

HARRY VEITCHII-Beautiful pink, large flower with waved petals. Mid to late season. Rather slow grower.

MAXWELL-Carmine red, mid-season, very hardy.

PERFECTION-Rose pink, large flower, mid-season.

PRAESTANTISSIMA-Carmine red, blooms a week earlier than Pride of Dorking. 
PRIDE OF MOBILE-Lovely watermelon pink. Flower very large, mid-season. Large leaves, very vigorous grower.

One of best Indica Azaleas.

PRIDE OF DORKING-Beautiful ceries of carmine, late, compact low grower.

\section{ORANGE RED}

COCCINEA MAJOR-Brilliant scarlet, early season, smalt leaves.

DAPHNE RED-Orange red, with purple spot on the throat Flower medium, very early, semi-weeping nature.

GLORY OF SUNNINGHILL-Large orange scarlet flower, late, compact grower.

MOSS POINT RED-Orange red with purple spots at the throat, mid-to-late season.

PRESIDENT CLAY-Orange red with purple spot at the throat. Bloom early.

STRAWBERRY RED-Red flower, early, slender, semi-weeping nature.

\section{SALMON}

DAPHNE SALMON-Medium flower with light salmon, early

DUC DE ROHAN-Medium size flower of lovely salmon pink. bloom early, leaves small, shinny green, compact.

DUKE OF WELLINGTON-Soft salmon, with darker center, mid-esason.

MOSS POINT LATE SALMON-Pretty salmon color, very large flower with widely waved petals. Very late, hardy vigorous grower.

WILLIAM BULL-Double flower of mud-red color, late, exceedingly vigorous and upright grower.

\section{LAVENDER-LILAC}

FORMOSA-Large lavender flower, mid-season, large leaves, most vigorous grower.

OMURASAKI-Large, dark lavender flower, hardy, mid-season

ORCHID LAVENDER-Medium flower of light orchid lavender pink, very early, upright growth.

PHOENICIA-Lavender pink, mid-season, low and compact. ROSY PURPUREA-Wine-red color. Late blooming.

VITATA FORTUNEI PURPLEANA-Separated from variegated kind, fine lavender pink, very early, upright open growth. 


\section{WHITE}

FIELDERS WHITE-Large white flower, early.

FLAG OF TRUCE-Mid-season, full double with waved petals.

INDICA ALBA-(Ledifolia Alba) Snow white large flower, early to middle season, very hardy.

LATERETIA ALBA-Medium size pure white flower, late small leaves, very compact growth.

\section{VARIEGATED}

CRITERION-White ground well malrked with wide pink stripes, late, compact.

INDICA ROSEA-(Ledifolia Rosea) Extra large flower of white with rose throat. Mid-season, upright.

IVERLANA-White ground marked with narrow, faint pink stripes, late, compact.

LADY EDITH-Variegated just like Criterion, but the flower slightly smaller and color of pink softer. Mid-season, compact.

VITATA FORTUNEI-White ground well striped with lavender pink. Very early. Upright, open grower.

\section{Price on Indica Azalea}

$\begin{array}{lrr}4-6 \text { inch C. T. } & 10 & 100 \\ 6-8 \text { inch C. T. } & .65 & \$ 5.00 \\ 4-6 \text { inch C. } 21 / 4 \text { inch pot } & .90 & 7.00 \\ 6-8 \text { inch C. TT., bushy, B\&B } & .75 & 6.00 \\ 8-10 \text { inch C. TT., bushy, B\&B } & 1.50 & 12.00 \\ 10-12 \text { inch C. TT., bushy, B\&B } & 2.00 & 17.50 \\ 12-18 \text { inch C. TTT., bushy, B\&B } \ldots & 2.50 & 22.50 \\ & \end{array}$

\section{HARDY JAPANESE AND KURUME AZALEA}

BENIGIRI-Bright deep pink, single, mid-season, upright.

BRIDE MAID-Bright salmon flower in large trusses. Midseason, low, compact.

CHRISTMAS CHEER-Pleasant Christmas red, flower small, hose-in-hose, early compact.

FLAME-Single large flower of brick dust red, very early, upright.

HEXE-(Firefly) Semi-double, medium size. Bright crimson, lowheaded, compact. Mid-season.

HINODEGI,RI-Most popular Japanese variety. Single, medium size, bright scarlet. Mid-season, dwarf and compact.

HINAMOYO-Medium size flower of soft pink, mid-season, upright. 
KIMUNAZOI-Very narrow petals of medium size flower of orange red, very late. Leaves very small and narrow, low head, compact.

MACRANTHA, SINGLE-Large flower of clear pink, very late, low head, compact.

MACRANTHA, DOUBLE-Double flower of large size with clear pink, very late also a few blossoms open through the summer. Compact but rather upright.

MACROSTEMON-Large, single salmon pink, late compact.

ORANGE BEAUTY-Good size flower of orange red color. Mid-season, compact.

PEACH BLOW-Pale shade of pink. Mid-season.

PINK PEARL-Cherry rose, shading lighter toward center, hose-in-hose. Mid-season, compact, but upright.

SNOW-Pure white, hose-in-hose, mid-season, low, compact.

SUNSTAR-Flower small, single dark rose pink with carmine stripes. Low compact.

YAEGIRI-Salmon red, hose-in-hose. Mid to late season. Compact, but upright.

Prices on Hardy Japanese and Karume Azaleas.

$\begin{array}{lrr} & 10 & 100 \\ \text { 3-5 inch C. T. } & \$ 65 & \$ 5.00 \\ \text { 3-5 inch C. T., 2 1-4 inch pot } & .90 & 7.00 \\ 5-6 \text { inch C. TT., bushy, B\&B } & 2.50 & 20.00 \\ \text { 6-8 inch C. TT., bushy, B\&B } & 3.00 & 25.00 \\ \text { 8-10 inch C. TTT., bushy, B\&B } & 4.00 & 35.00 \\ 10-12 \text { inch C. TTT., bushy, B\&B } & 5.50 & 50.00 \\ 12-15 \text { inch C. TTT., bushy, B\&B } & 8.00 & 75.00 \\ \text { (We can supply last two sizes in HINODEGIRI, FLAME, } \\ \text { HINAMOYO, SNOW and PINK PEARL. }\end{array}$

BERBERIS ATROCARPA (Evergreen Berberry)

4-6 inch C. T.

$\$ .65 \$ 5.00$

8-12 inch C. T.

12-18 inch C. TT.
1.00

10.00

15-18 inch $B \& B$

1.25

.50

BOTTLE BRUSH (Callistemen)

18-24 inch 4-inch pot _ _ _ _..... .25

2-3 feet, 6-inch pot _ _ $\quad .50$

\section{BUXUS JAPONICA (Japan Boxwood)}

3-5 inch C. $T$.

10-12 inch C. TT., bushy

12-15 inch C. TT., bushy

10-12 inch $B \& B$

12-18 inch $B \& B$ 
BUXUS SEMPERVIRENS (English Boxwood)

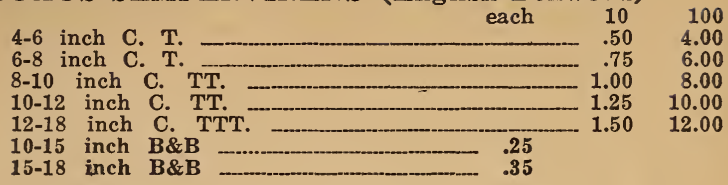

\section{CAMELLIA JAPONICA}

Plants offered here are all pot-grown, as we have found it is far superior to the plants from the ground and poorly balled and burlaped. . Plants from pots can also be safely removed any time of the year.

\section{Group No. 1.}

ABBY WILDER, PINK-Very double peony-form. Generally pink but sometimes marked with white. (Abby Wilder generally pink and white variegated. This strain must be propagated from pink branch).

CHANDLERII ELEGANS-Semi-double to double, flower very large, pink variegated with white, somewhat peony form mixed with yellow stamens.

COUNTES OF ORKNEY-Very double, pink striped on white ground. Slow grower but it blooms very young and small. Best for pot culture.

DUC DE BRETOGNE-Very double, pretty rose, lighter color toward center. Slow but very compact grower.

HERME-Semi-double, very large flower variegated pink with white generally white margin of the petals.

LADY HUME'S BLUSH-Medium size flower very double and delicate flesh pink.

OTOME-Very much alike to Pink Perfection. Only flower slightly larger so with petals. Blooms later than Pink Perfection.

PROF. CARGENT-Very full double, peony form, dark red.

REINE DE FLAURES-Rich vermillion red flaked with white

\section{Prices on Group No. 1}

3 inch pot, 8-12 inch

except Chandlerii Elegans, Countes of

Orkney and Duc De Bretogne which

are very slow grower. 6-8 inch.

$\$ 6.00 \$ 50.00$

21/2 inch pot, 6-8 inch, except slower

growers, which are 4-6 inch

Strong rooted cutting

$2.00 \quad 17.50$ 
Group No. 2

ALBA PLENO-Large flower, very double, pure white, early. Best white.

AMERICANA-Soft pink striped with crimson.

ANNA FROST-Large, semi-double, pink color like American Beauty Rose.

ARNALDA DE BRESEIA-Double, deep pink, with strips of white in center of the petals.

BROOKLYANA-Very double, medium size flower, deep pink mottled with white.

CALEB COPE-Double deep flesh pink.

CANDIDISSIMA-Medium flower, pure white, small petals wrapped each other symmetrically forming star shape. Late bloomer.

CHEERFULNESS-Double, bright pink, medium size, early bloomer.

DERBEYANA-Semi-double to double, dark red or vermillion red, large flower with large petals. Yellow stamens mixed.

GUILBEAU'S PINK-Large, semi-double to double, Rose pink, shape of the flower resembles to Herme.

LADY SAUMEREZ-Semi-double, cherry-red, early bloomer.

LADY VANSITTI-Semi-double, large flower, most desirable color of red.

MADONA-Double flower, flesh color, some times spotted with darker color.

MM. PICOULINE-Crimpled petals or peony form, bright red.

MATHOTIANA ALBA-Flower very large of shape like cup on saucer. Pure white but sometimes striped with slight pink, late bloomer.

MARGUERITE GOUILLON-(Duke de Orleans) Peony form, creamy white, ground flaked and dotted with rosy pink.

OPELOUSAS' PEONY-Large peony-form. Light pink occasionally spotted with white.

PINK PERFECTION-Very popular variety, flower medium, very full double with immense small petals symmetrically arranged. Lovely shell pink, early and free bloomer.

PRESTON ROSE-Flower medium, full double, peony-form, pretty pink, occasionally with a little white spots.

PURITY-Pure white, double, but rather open center, easy to cultivate.

SACCO-Medium flower, bright rose pink.

Prices on Group No. 2

4 inch pot, 12-18 inch

3 inch pot, 8-12 inch height __ 3.50

$21 / 2$ inch pot, 6-8 inch 


\section{Group No. 3}

CAMBELLI-Large flower, deep rose, very double.

DARSII-Medium flower, semi-double to double with yellow stamens. Admirable bright red, early and free bloomer.

JARVIS RED-Semi-double to double, dark vermillion, petals large, somewhat crinkled, yellow stamens mixed. Mid-season.

ORIENT-Flower small, dark red, peony form, resembles very much to Prof. Sargent, but size small.

SARAH FROST-Rosy crimson, very double, mid-season, free bloomer, hardy.

ALL UNNAMED VARIETIES (will be named soon as confirmed) which sold by color.

\section{Prices on Group No. 3}

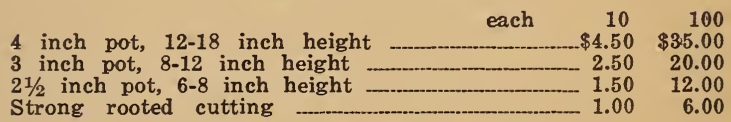

\section{Prices on Specimen Plants}

\section{DOUBLE RED.}

2-3 feet, very bushy and full, B\&B 2.00

3-4 feet, very bushy and full, $B \& B$ …. 3.00

4-5 feet, extra bushy and full, B\&B _.... 4.00

\section{CAMELLIA JAPONICA SEEDLING}
4-6 inch
(S)
.60
4.00
6-8 inch (S)

\section{CAMELLIA SASANQUA}

\section{Single white( pink tinted at edge of petals).}

Single pink, (shell pink, large flower).

Semi-double white (white with pink tinted, more compact grower, variegated leaves).

Double white, Mine-no-yuki, (pure white, very double, peonyform).
6-8 inch C. T.
$8-12$ inch $\mathrm{C}$. $\mathrm{T}$.
1.00
1.25
12-18 inch C. TT. (no double white)
18-24 inch $B \& B$
$2.00 \quad 15.00$
2-3 feet $B \& B$
1.00

\section{CINNAMOMUM CAMPHORA (Camphor Tree)}

3-4 feet, Bare-root, defoliated ___ .40

4-5 feet, Bare-root, defoliated ……...... $\quad .50$

5-7 feet, Bare-root, defoliated …........................

\section{CLEYERA JAPONICA}

4-6 inch $C$. $T$.

6-8 inch C. $\mathrm{T}$.

15-18 inch $B \& B$

18-24 inch $B \& B$ 


\section{TWO WILD SAGES, Witl}

Being Believed by Us the $B$

\begin{tabular}{|c|}
\hline $\begin{array}{l}\text { RED SAGE } \\
\text { podium Coccineam) }\end{array}$ \\
\hline $\begin{array}{l}\text { This is one of the most prospective native } \\
\text { plants of South Alabama. It grows in poorest } \\
\text { sandy or rocky soil. A slender, sparingly } \\
\text { branched shrub of two or three feet tall with } \\
\text { small evergreen leaves and horizontial, tubu- } \\
\text { lar, bright red flower blooming all the summer } \\
\text { and the fall until just before Christmas, es- } \\
\text { pecially at autumn, even young plant of a few } \\
\text { inches, covered with mass of red flowers. In } \\
\text { addition to its attractive appearance, it has } \\
\text { pleasant odor which might possibly be utilized } \\
\text { in perfumery No cold spell ever injured this } \\
\text { plant down here, it may stand near zero } \\
\text { weather, probably lower. No doubt this is } \\
\text { one of most suitable plants for Southern rock } \\
\text { garden, also it will be very valuable pot plant. }\end{array}$ \\
\hline Special Trial Offer. \\
\hline $\begin{array}{l}25 \text { plants } 8-10 \text { inch (collect) for } \\
\text { (Large quantity upon application) }\end{array} \$ 2.00$ \\
\hline
\end{tabular}

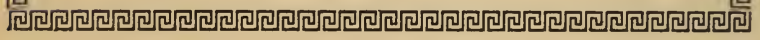




\section{Prospect of a Great Fu ture}

\section{of Our Now Introductions}

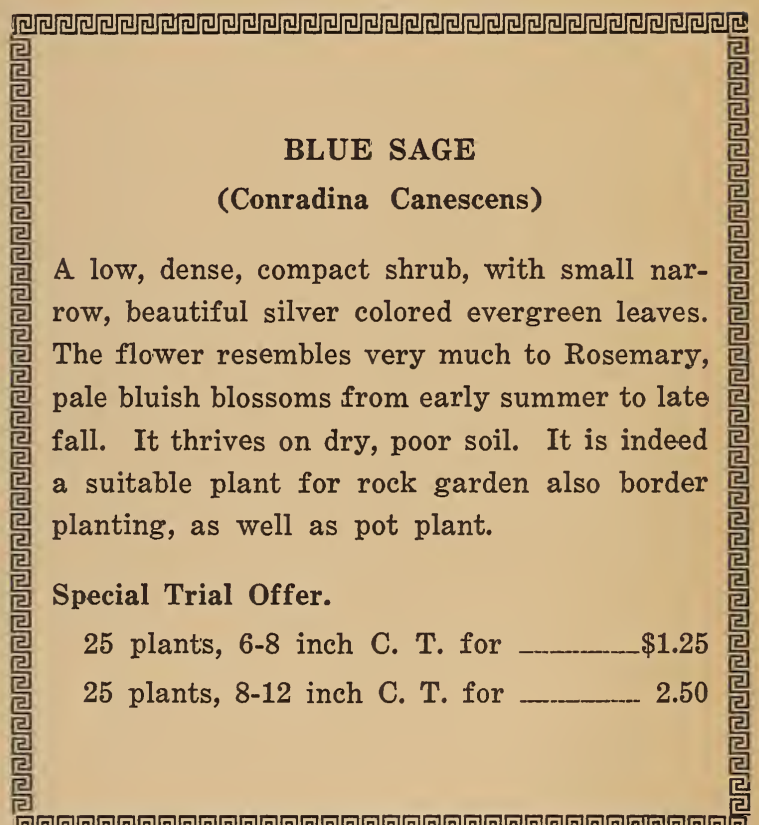

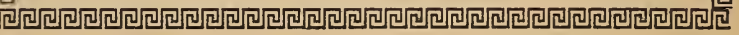


ELAEGNUS PUNGEN VARIEGATA

(Yellow Edge)

\section{ELAEGNUS PUNGEN MACULATA}

(Yellow Center)

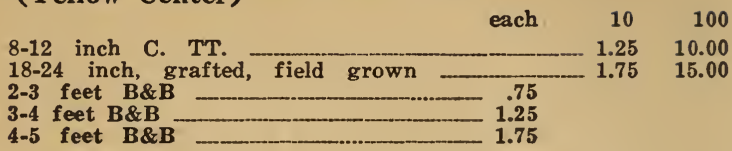

\section{ELAEAGNUS PUNGEN FRUITLANDI}

8-12 inch $\mathrm{C}$. $\mathrm{T}$.

12-18 inch C. TT.

10.00

18-24 inch, grafted, field grown

2-3 feet $B \& B$

3-4 feet $B \& B$

12.00

4-5 feet $B \& B$

\section{ELAEAGNUS PUNGEN REFLEXA}

(Semi-weeping)

\section{ELAEAGNUS PUNGEN SIMONI}

12-18 inch, grafted, field grown

18-24 inch, grafted, field grown

2-3 feet B\&B

3-4 feet B\&B

\section{EUONYMUS CARRIERI}

8-10 inch C. T. .65

\section{EUONYMUS PATENS}

6-8 inch $\mathrm{C}$. $\mathrm{T}$.

EUONYMUS JAPONICA ALBO-MARGINATA

4-6 inch $C$. $T$.

EUONYMUS JAPONICA MICROPHYLLA (Miniature Euonymus)

2-4 inch C. $T$.

4-6 inch C. T.

.60

6.00

(For Euonymus Radicans and others see under heading "Vine.)

\section{FATISIA JAPONICA}

8-10 inch $\mathrm{S}$. TT.

1.50

10-12 inch $\mathrm{S}$. TT.

12-15 inch $\mathrm{S}$. TT.

FEIJOA SELLOWIANA (Pineapple Guava) 


\section{GARDENIA FLORIDA}

(Cape Jasmine)

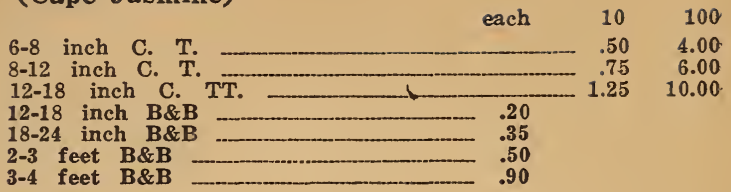

GARDENIA RADICANS

(Creeping Cape Jasmine)

$\begin{array}{lllrr}4-6 \text { inch } \text { C. T. } & .50 & 4.00 \\ 6-8 \text { inch } & \text { C. T. T. } & .75 & 6.00 \\ 8-10 \text { inch } \text { C. TT. } & 1.00 & 8.00 \\ 10-12 \text { inch C. TT. } & 1.25 & 10.00 \\ 12-15 \text { inch spread B\&B } & & \\ 15-18 \text { inch spread B\&B } & .35 & & \end{array}$

GARDENIA STRICTA NANA

(Dwarf Cape Jasmine)

4-6 inch C. T. T__ $\quad .50 \quad 4.00$

6-8 inch C. T.

GARDENIA VIETCHI

(Winter Blooming Cape Jasmine)

4-6 inch C., $2 \frac{1}{8}$ inch pot $1.00 \quad 8.00$

\section{ILEX CASSINE (Dahoon Holly)}

4-6 inch C. T. _-

6-8 inch C. T.

8-12 inch C. T. 1.25

\section{ILEX CORNUTA (Chinese Holly)}

4-6 inch $C$. $T$.

6-8 inch C. T.

8-12 inch C. TT.

12-18 inch C. TT.

\section{ILEX CRENATA (Japan Dwarf Holly)}

2-4 inch $S$.

4-6 inch $S$.

\section{ILEX CRENATA ROTANDIFOLIA}

\section{(Round Leaved Japan Dwarf Holly)}

4-6 inch

6-8 inch

8-10 inch $\mathrm{C}$.

10-12 inch C. TT.

12-15 inch, very bushy, B\&B

15-18 inch, very bushy, B\&B 


\section{ILEX INTEGRA}

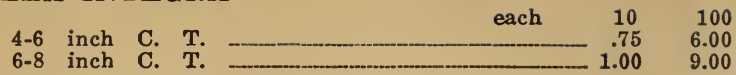

ILEX LATIFOLIA (Big-Leaved Holly)

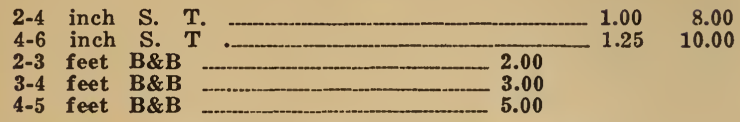

\section{ILEX MYRTIFOLIA (Myrtle-Leaved Holly)}

$2-4$ inch C. T. …_.

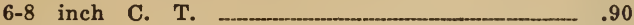

ILEX OPACA FEMINA

(American Holly Berried Kind)

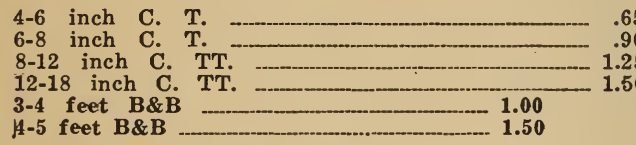

ILEX VOMITORIA FEMINA (Berry-Bearing Yupon)

$\begin{array}{lllrr}2-4 \text { inch C. } & \text { T. } & .65 & 5.00 \\ 6-8 \text { inch } & \text { C. } & .90 & 7.00 \\ 8-12 \text { inch } & \text { C. } & \text { TT. } & 1.25 & 9.00 \\ 12-15 \text { inch C. TT. } & 1.50 & 12.00\end{array}$

\section{ILLUCIUM ANISATUM}

12-18 inch C. TT.

3-4 feet B\&B

\section{JASMINUM FLORIDUM}

8-12 inch C. T.

12-18 inch C. TT.

\section{JASMINUM HUMILE}

8-12 inch C. $\mathrm{T}$.

12-18 inch C. TT.

12-18 inch C.

\section{JASMINUM PRIMULINUM}

8-12 inch C. T.

12-18 inch

C. TT

\section{LAUROCERASUS OFFICINALIS}

(English Laurel)

6-8 inch C. T.

8-12 inch C. TT. 
LIGUSTRUM CORIACEUM (Dwarf Privet)

2-4 inch C. $\mathrm{T}$.

each

4-6 inch

C. $\mathrm{T}$.

.75

LIGUSTRUM JAPONICUM AUREUM

(Golden Japan Privet)

LIGUSTRUM JAPONICUM MACROPHYLLUM (Big-leaf Japan Privet)

\section{LIGUSTRUM JAPONICUM EXCELSUM}

SUPERBUM (Variegated Japan Privet)

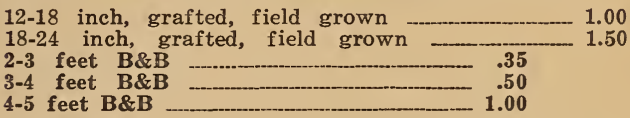

LIGUSTRUM CILIATUM VARIEGATUM (Iwaki Privet)

LIGUSTRUM LUCIDUM COMPACTUM

(True Waxy Privet)

\section{LIGUSTRUM LUCIDUM PYRAMIDALIS} GRACILIS

(Glossy upright Privet)

\section{LIGUSTRUM LUCIDUM REPENDA}

(Crinkled-Leaf Privet)

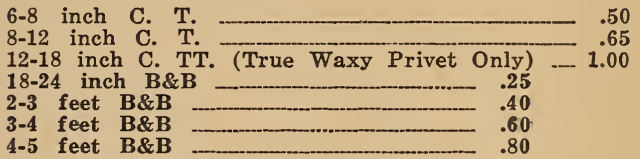

\section{LIGUSTRUM IONANDUM}

6-8 inch C. T.

5.00

8-12 inch

C. T.

MAGNOLIA FUSCATA

4-6 inch C. T., (Spring delivery only) _- $.75 \quad 6.00$

6-8 inch C. T.

8-12 inch C. T'T.

12-18 inch $B \& B$

18-24 inch $B \& B$

2-3 feet B\&B

3-4 feet $B \& B$

\section{MAGNOLIA GRANDIFLORA}

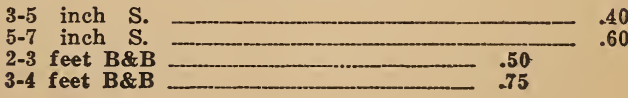




$\begin{array}{lrlrrr}6-8 \text { inch } & \text { S. TT. } & \text { each } & 10 & 100 \\ 8-10 \text { inch } & \text { S. TT. TT. } & 1.00 & 8.00 \\ 10-12 \text { inch } & \text { S. TT. TT. } & 1.25 & 10.00 \\ - & 1.75 & 15.00\end{array}$

\section{MALPIGHIA GLABRA (Barbadoe Cherry)}

6-8 inch C. $T$.

8-12 inch C. TT.

\section{NANDINA DOMESTICA}

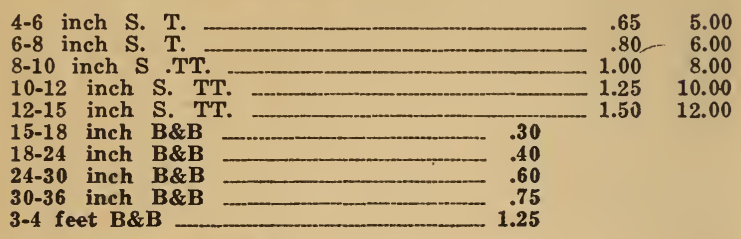

\section{NERUM (Oleander)}

White, Pink, Buff and Red.

2-3 feet B\&B

3-4 feet $B \& B$ .45 .75

OSMANTHUS AQUIFOLIUM (Holly Olive) OSMANTHUS FORTUNEI (Tea Olive) OSMANTHUS FRAGRANCE (Sweet Olive)

4-6 inch C. T.

6-8 inch C. T.

$8-12$ inch C. TT.

12-18 inch C. TT.

18-24 inch B\&B

2-3 feet $B \& B$

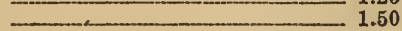

.50

.75

(Last two sizes Tea and Sweet Olive)

\section{PHOTINIA GLABRA}

6-8 inch C. T.

8-12 inch C. T.

12-18 inch C. TT.

8-12 inch C., $2 \frac{1 / 2}{2}$ inch pot

8.00

12-18 inch C., 3 inch pot

7.00

18-24 inch $B \& B$

3-4 feet $B$ \& $B$

4-5 feet $B \& B$ .75

\section{PHOTINIA SERRULATA}

4-6 inch C. T. (Spring delivery only) 


\section{PIERIS JAPONICA}

each $\quad 10 \quad 100$

3-5 inch C., 21/4 inch pot ___ $1.00 \quad 8.00$

12-15 inch $B \& B$ _

15-18 inch $B \& B$ _ 1.00

18-24 inch $B \& B$ _- 1.25

\section{PITTOSPORUM TOBIRA}

6-8 inch C. $\mathrm{T}$.

8-12 inch C. T.

7.00

12-18 inch C. TT.

18-24 inch $B \& B$

10.00

2-3 feet $B \& B$

.75

\section{PITTOSPORUM TOBIRA VARIEGATA}

4-6 inch $C$. $T$.

6-8 inch C. T.

8-12 inch C. TT.

12-15 inch spread $\bar{B} \& B$

PYRACANTHA LALANDI (Yellow Berried)

PYRACANTHA FORMOSANA (Scarlet)

PYRACANTHA YUNNANENSIS (Coral)

PYRACANTHA GIBBSII (Orange red)

6-8 inch C. T.

8-12 inch C. $T$.

18-24 inch $B \& B$

2-3 feet $B \& B$

\section{RAPHIOLEPIS INDICA}

\section{RAPHIOLEPIS JAPONICA}

8-12 inch $\mathrm{S}$. $\mathrm{T}$.

1.00

12-18 inch $\mathrm{S}$. $\mathrm{T}$.

15-18 inch $B \& B$

$1.25 \quad 10.00$

18-24 inch $B \& B$

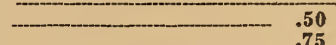

\section{RAPHIOLEPIS DELACOURII}

A hybrid between R. Indica and R. Salicifolia. Foliage, intermediate, shinny green, upright grower and large panicles of blushed flower blooms at mid-winter.

4-6 inch C. T. (Spring delivery only) ___ $1.00 \quad 8.00$

12-18 inch C. TT.

$1.75 \quad 15.00$

\section{SERISSA FOETIDA}

12-18 inch C. TT.

18-24 inch B\&B

.75

\section{VIBURNUM MACROPHYLUM}

(Southern Sweet Viburnum) 


\section{VIBURNUM ODORATISSIMUM \\ (Japanese Glossy Viburnum)}

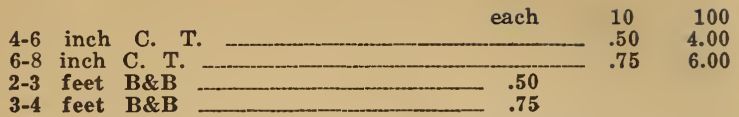

\section{VIBURNUM SUSPENSUM (Sandankwa)}

\section{VIBURNUM TINUS}

4-6 inch C. T.

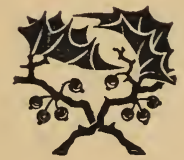




\section{DECIDUDOUS TREES AND SHRUBS}

\section{ALBIZZIA JULIBLISSIN (Mimosa Tree)}

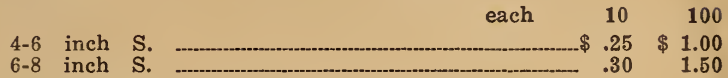

\section{ALEURITES (Chinese Tung-oil Tree)}

3-4 feet S. T.

4-5 feet $S$. T.

.15

5-7 feet S. T.

\section{ALTHEA}

Varieties.

Anemonaeflorus (Double Rose)

Areens, (Double violet)

Boul de Feu (Purplish red)

Jeanne de Are (Double pure white)

Lady Stanley (Semi-double white red center)

Lucy (Double Red)

Purpurea (Semi-double purple)

Rubis (Single red)

Totus Albus (Single white)

12-18 inch C. Field

18-24 inch C. Field

2-3 feet

3-4 feet

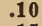

.15

\section{AMYGDALUS (Flowering Peach)}

Double red, double pink and double white.

12-18 inch, grafted on plum stock

BUDDLEIA LINDLEYANA

(Ever-blooming Butterfly Bush)

6-8 inch C. T.

.50

BUDDLEIA (Magnifica)

No. 1

Medium

\section{CALYCANTHUS FLORIDUS}

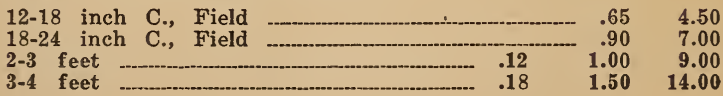


CORNUS FLORIDUS (White Flowering Dogwood)

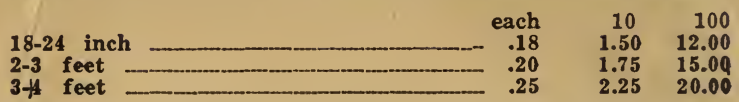

,CORNUS FLORIDA RUBRA (Pink Flowering Dogwood)

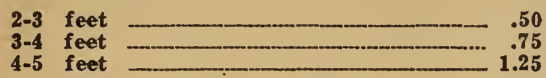

CYDONIA JAPONICA ROSEA

12-15 inch

\section{CYTISUS SCOPARIUM (Scotch Broom)}

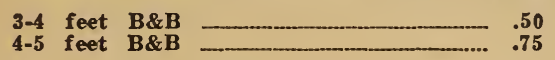

FORSYTHIA

Varieties-Intermedia, Suspensa, Fortunei and Spectabilis.

$\begin{array}{llllll}3-4 & \text { feet } \\ 4-5 & \text { feet } & & .20 & 1.50 & 12.00 \\ & .25 & 2.00 & 18.00\end{array}$

HYDRANGTA P. G.

$12-18$ inch

18-24 inch _-___- $1.25 \quad 9.00$

IDESIA POLYCARPA

Desirable garden tree, growing in height about 20 feet, leaves in heart shape, turning yellow to scarlet at Autumn. Clustered red berries in Winter, exceptionally attractive.

Rapid grower.

$6-8$ inch S. $\quad .75 \quad 5.00$

8-12 inch S. W-

LAGERSTROMIA INDICA (Crape Myrtle)

Crimson, white and lavender.

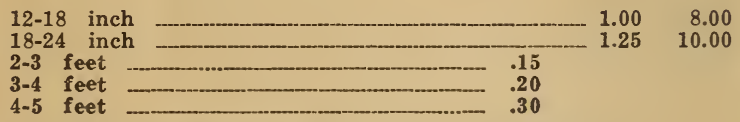

LAGERSTROMIA INDICA (Crape Myrtle)

Crimson (very dwarf) Flesh color and deep purple.

2-4 inch C. T. $1.00 \quad 8.00$

LESPEDEZA FORMOSA (Pink Flower)

12-18 inch $\mathrm{S}$.

18-24 inch $\mathrm{S}$.

LESPEDEZA JAPONICA (White)

Small division, 3 to 5 eyes

Medium division, 5 to 10 eyes .65 


\section{LIGUSTRUM AMURENSE}

(Amoor River Privet North)

$\begin{array}{cccc} & \text { each } & 10 & 100 \\ 12-18 \text { inch } & .35 & 1.50 \\ 18-24 \text { inch } & .50 & 2.50\end{array}$

\section{LIGUSTRUM OVALIFOLIUM}

(California Privet)

$12-18$ inch

$18-24$ inch _.

\section{LIGUSTRUM REGELAS}

12-18 inch

18-24 inch

\section{LIGUSTRUM IBOLIUM}

12-18 inch

LONICERA MORROWI

12-18 inch

\section{MAGNOLIA LILIFLORA}

(Purple outside and White inside)

4-6 inch C., 21/8 inch pot

8-12 inch C. TT.

$12-18$ inch C. TT.

2-3 feet $B \& B$

3-4 feet $B \& B$

4-5 feet $B \& B$

1.00

2.50

MAGNOLIA SOULANGEANA NIGRA

(Dark Purple)

3-5 inch $2 \frac{1}{8}$ inch pot

2.00

15.00

MAGNOLIA SOULANGEANA (Pinkish Flower)

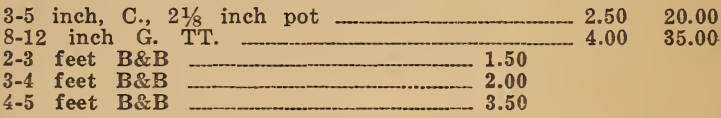

MALUS EXCELENCA (Weeping)

MALUS FLORIBANDA (Single Pink)

MALUS NIEDZWETZKYANA (Red)

MALUS SPECTABILIS (Double Pink)

12-18 inch

18-24 inch 


\section{PRUNUS PISSARDI (Purple-leaf Plum)}

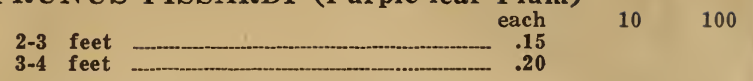

\section{SPIREA ANTHONY WATERER}

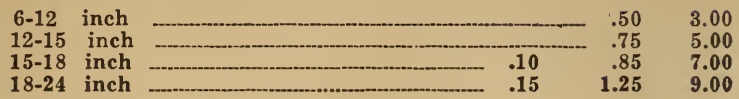

SPIREA ARGUTA

$\begin{array}{rrrrrr}2-3 & \text { feet } & & .10 & .85 & 7.50 \\ 3-4 & \text { feet } & .15 & 1.25 & 10.00\end{array}$

\section{SPIREA THUNBERGIA}

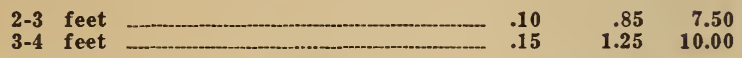

\section{SPIREA VAN HOUTTI}

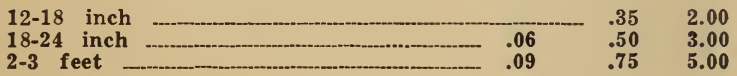

WEIGELIA

Eva Rathka (Red)

Floribunda (Crimston)

12-18 inch

18-24 inch

2-3 feet

\section{WEIGELIA}

\section{Rosea (Rosy Pink)}

Nana Variegate (Variegated Leaf)

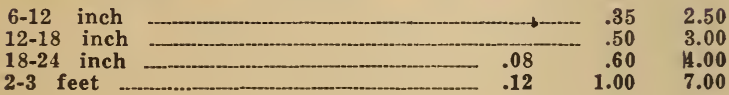




\section{VINE AND CLIMBER}

\section{BIGNONIA SPECIOSA}

Evergreen vine with large purple trumpet shaped flower.

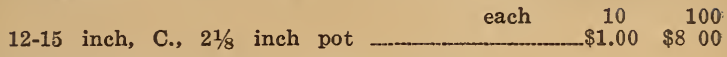

BOUGAINVILLEA (Crimson Lake)

6-8 inch, $2 \frac{1}{2}$ inch pot

$1.25 \quad 10.00$

EUONYMUS RADICANS (Winter Creeper)

8-10 inch C. T.

EUONYMUS RADICANS VARIEGATA

6-8 inch C., $2 \frac{1}{4}$ inch pot $1.00 \quad 8.00$

EUONYMUS KIWENSIS (Baby Winter Creeper)

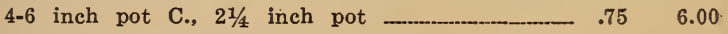
FICUS REPENS (Climbing Fig)

6-8 inch C., 21/4 inch pot __.____ $75 \quad 6.00$

HEDERA HELIX (English Ivy)

(Large and small leaf)

8-12 inch, $2 \frac{1}{4}$ inch pot

KADZURA JAPONICA

Evergreen vine bore red berries.

8-12 inch C., $2 \frac{1}{4}$ inch pot

MICHELLA REPENS (Partridge Berry)

$2 \frac{1}{2}$ inch pot .65

MILLETIA JAPONICA (Formosa Wisteria)

Wisteria like deep purple flower blooms during summer months.

8-12 inch C. T., in field

12-18 inch, C. T. infield

$1.75 \quad 15.00$

STAUNTONIA HEXAPHYLLA

(Evergreen Akebia)

2-4 inch $S$.

4-6 inch $\mathrm{S}$.

1.2510 .00

$1.50 \quad 12.00$

TRACHELOSPERUM JASMINOIDES

(Star Jasmine)

8-12 inch, C., $2 \frac{1}{2}$ inch pot $.75 \quad 6.00$ 
WISTERIA MULTIJUGA (Purple, Long Cluster)

2-year, grafted

WISTERIA CHINENSIS ALBA

(Large White Flowers)

2-year, grafted .40

WISTERIA MULTIJUGA ROSEA (Pink)

2-year, grafted

Extra large 3-4 year old grafted

.50

each
.30

100

PALM, GRASS, and MISCELLANEOUS

\section{CHAMEROPS EXCELSA (Windmill Palm)}

18-24 inch B\&B _. 75

$2-3$ feet $B \& B$

\section{COCOS AUSTRALIS (Pindo Palm)}

8-12 inch S. ___ $\quad .50 \quad 4.00$

18-24 inch, S., 3-year old ___ $1.25 \quad 10.00$

(We have fine large specimens, write for price).

\section{COCOS ERIOSPATHA}

Hardiest palm of this sort. Resembles very much to Cocos Australis.

8-12 inch, S., 1-year .65

YUCCA GLORIOSA

4-6 inch, S., 2-year old .65

OPHIOPOGAN JAPONICA

(Japanese Blue Grass)

Small division

Medium division

OPHIOPOGAN JUBURAN AUREA VARIEGATA

Fine variegated plants for border as well as house plant. Medium clump $3.00 \quad 25.00$

CORTADERIA ARGENTEA (Pampas Grass)

Silver and pink.

Small division

Medium division .75 1.25

MISCANTHUS JAPONICA VARIEGATA

Well variegated fine plant.

$2 \frac{1}{2}$ inch pot 1.00 


\section{MISCANTHUS JAPONICA ZEBRINA}

Small division

Medium division

\section{BAMBOO ARGENTEA}

Best Ornamental Bamboo.

\section{BAMBOO FALCATA} Dwarf Bamboo.

Small division

Medium llivision

\section{LIATRIS (Blazing Star)}

Lavender and white.

Blooming bulb

POTHOE

Beautiful vine with variegated leaves. Stand dry, heat, one of best house plants.

$21 / 2$ inch pot

\section{SAXIFRAGAS}

$21 / 2$ inch pot

Well rooted small plants

\section{VINCA VARIEGATA}

$2 \frac{1}{2}$ inch pot

\section{SARRACENIA (Pitcher Plant)}

S. Flava. (Yellow leaves and yellow flower)

S. Drummondii (Red leaves and red flower)

S. Purpurea (very dwarf, reddish leaves and pink flower).

S. Psittacina (Very dwarf, green leaves and yellow flower)

Good plants (collected)

$.75 \quad 5.00$

\section{FRUIT AND NUT TREES}

PECAN (Stewart and Success)

3-4 feet

4-5 feet

5-7 feet

\section{JAPANESE PERSIMMON}

\section{(Tanenashio and Fuyu)}

3-4 feet

\section{PLUM (Excelsa)}

Grafted on Plum Stock.

2-3 feet

5-7 feet 




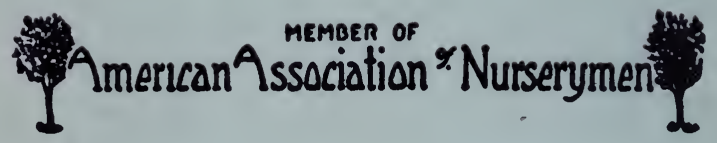

